\title{
Soziale Netze - neue Impulse zum Datenschutz
}

Datenschutz und Web 2.0 sind derzeit ein heißes Eisen, nicht zuletzt weil die „sozialen Netze“ aktuell riesige Experimentierfelder sind. Sie treffen zwar die Bedürfnisse der Informationsgesellschaft, fragen aber oft nicht nach den rechtlichen Rahmenbedingungen oder Risiken. Die Möglichkeit, die virtuelle Gemeinschaft mit zu gestalten, ist reizvoll, die hohen Nutzerzahlen sprechen hier für sich. Beachtenswert ist in diesem Zusammenhang aber auch, dass viele Nutzer die Wirkungen ihres eigenen Handelns in den Netzwerken kritisch verfolgen und aktiv an der Gestaltung der Regeln der Betreiber zum Umgang mit Daten und Profilen mitwirken. Diese Chance ist in vielen anderen Anwendungsbereichen nicht gegeben.

Aber selbst Datenschutzstandards, die in Bezug auf einfachere Sachverhalte längst veraltet sind, werden in der Welt der sozialen Netzwerke oft nicht eingehalten. Allerdings haben die Nutzer hier nun die Chance, dem sozialen Verhalten in der virtuellen Welt kulturelle Qualitäten im Umgang mit Daten hinzuzufügen und daraus auch abzuleiten, welche neuen Impulse das Datenschutzrecht daraus gewinnen kann.

\section{Neue Wege zum Datenschutz 2.0}

Insbesondere zwei Aspekte, die sich aus der neuen Rolle der Nutzer als Mitgestalter sozialer Netze ergeben, sind für eine Modernisierung des Datenschutzrechts bedenkenswert:

- Wie werden der Anspruch und die Grenzen der informationellen Selbstbestimmung im Hinblick auf Eigenverantwortung, Vertrauensanspruch an andere Teilnehmer und den Netzwerkbetreiber justiert?

- Wohin wird sich zukünftig - vor Allem aus Sicht der aktiven Nutzer - das „Schutzgut“ Privatsphäre entwickeln? Darüber hinaus ist es selbstverständlich notwendig, die Risiken, die sich aus den Geschäftsmodellen der Betreiber sozialer Netzwerke für die Daten der Nutzer ergeben, zu beobachten und dabei generell für Gefahren und Angriffe von Innen und Außen zu sensibilisieren.

Im Schnitt ist nach aktuellen Zahlen knapp jeder zehnte Deutscher Mitglied in einem der VZs. Dieser Trend bringt allerdings auch erhebliche Risiken mit sich und hat vor allem in der jüngeren Vergangenheit auch immer wieder für negative Schlagzeilen in den Medien gesorgt. Einer der Gründe hierfür ist, dass soziale Netzwerke und deren Profile immer mehr zur Zielscheibe Krimineller werden, die das Potential der riesigen Datenmengen erkannt haben. Denn für persönliche Daten existieren zahlreiche Missbrauchsmöglichkeiten: Angefangen beim Identitätsdiebstahl und anschließendem -missbrauch, über rassistische Anfeindungen bis hin zum Cyber-Stalking bieten soziale Netzwerke einen hervorragenden Nährboden für kriminelle Machenschaften.

Darüber hinaus lassen sich schließlich mit Hilfe der Korrelation von Profildaten auch rasterfahndungsähnliche Methoden umsetzen. Die Veröffentlichung von persönlichen Daten birgt nicht zuletzt auch ein großes ökonomisches Potential - gegenüber den vorher beschriebenen Folgen aber wohl ein eher harmloses Problem.

\section{Von Crawling und Profilkorrelationen}

Es ist daher keinesfalls unangebracht, auf die Gefahren einer übertriebenen Nutzung der sozialen Netzwerke hinzuweisen. Gerade bei der der jüngeren Generation werden der Datenschutz und das Missbrauchspotential oftmals unterschätzt, Daten wie das Geburtsdatum oder die politische Gesinnung werden bedenkenlos veröffentlicht.

Nutzer sozialer Netzwerke liegen aber völlig falsch, wenn sie annehmen, dass durch die Veröffentlichung von Bruchteilen ihres vollständigen Profils auf verschiedenen Netzwerk-Plattformen ein Missbrauch durch Dritte verhindert werden könnte. Ein Angreifer kann zunächst im großen Stil identitätsbezogene Profilinformationen und zugehörige -relationen aus mehreren sozialen Netzwerken sammeln, weil etwa Captchas nur unzureichend gegen heutige Angriffstechnologien schützen.

Im Anschluss daran kann er versuchen, bereits bestehende Profilinformationen durch Korrelationen anzureichern. Diese ergeben sich dann etwa durch Über- einstimmungen oder Ähnlichkeiten verschiedener Attribute und aufeinander verweisenden Relationen.

Internetnutzer sind nun oft in mehreren sozialen Netzwerken aktiv: Auf einer Plattform speichern sie ihre persönlichen Bilder, auf einer anderen pflegen sie persönliche Weblogs und auf einer dritten verwalten sie Kontakte zu Freunden, Bekannten und Arbeitskollegen. Durch Ähnlichkeiten der dabei verwendeten Profile kann man nun Schlüsse auf die reale Person hinter den ggf. auch verschiedenen virtuellen Identitäten ziehen.

\section{Datenschutz: Neubestimmung kann Krise verhindern}

Die Nutzung sozialer Netzwerke ist mittlerweile für viele Menschen aus dem Alltag nicht mehr wegzudenken. Der fade Beigeschmack der Intransparenz dieser Plattformen wird durch die vielen offensichtlichen Vorteile oft verdrängt, Nutzer geben ihre Daten "gerne“ preis, man hat ja nichts zu verbergen.

Tatsächlich hat der derzeitige Nutzer sozialer Netzwerke aber keine Kenntnis darüber, wie mit seinen persönlichen $\mathrm{Da}$ ten umgegangen wird. Aktuell verhalten sich soziale Netzwerke eher wie große Blackboxen, in die Daten eingespeist werden. Was mit diesen dann passiert, das weiß niemand so ganz genau, eine Kontrolle durch den Nutzer ist in den meisten Fällen nicht möglich.

Neben einer entsprechenden Medienkompetenz, die den Nutzer dazu befähigt, die langfristigen Auswirkungen zu freigiebiger Datenpreisgabe einzuschätzen, fehlt es aber auch noch an einem entsprechenden Rechtsbewusstsein und einer Kultur der ,verantwortungsvollen“ Nutzung der Daten sozialer Netzwerke.

Das gilt aber nicht nur für die „bösen“ Betreiber, sondern für jeden einzelnen Nutzer. Im Web 2.0 gibt es nämlich kein echtes Opfer-Täter-Prinzip mehr. Hier kann auch jeder Anwender (ungewollt) zum Täter werden. Schließlich geben gerade die Anwender die Daten und Profile ihrer „Freunde" oft ungefragt weiter und nicht immer nur der Betreiber des sozialen Netzes. 